\title{
The frozen elephant trunk technique in acute and chronic aortic dissection: intraoperative setting and patient selection are key to success
}

\author{
Tim Berger ${ }^{1,2}$, Martin Czerny ${ }^{1,2}$ \\ ${ }^{1}$ Department of Cardiovascular Surgery, University Heart Centre Freiburg, Freiburg, Germany; ${ }^{2}$ Faculty of Medicine, University of Freiburg, \\ Freiburg, Germany \\ Correspondence to: Tim Berger, MD. University Heart Centre Freiburg, Südring 15, 79189 Bad Krozingen, Freiburg, Germany. \\ Email: tim.berger@universitaets-herzzentrum.de.
}

Submitted Sep 02, 2019. Accepted for publication Oct 10, 2019.

doi: 10.21037/acs-2019-fet-10

View this article at: http://dx.doi.org/10.21037/acs-2019-fet-10

\section{Intra-operative settings}

The frozen elephant trunk (FET) technique is still challenging, even in high volume aortic centers. Therefore, adequate intra-operative settings are mandatory for an optimal clinical outcome. The use of cerebrospinal fluid drainage is not routinely required, as spinal cord injury could be effectively reduced to a minimum by using prostheses with the shortest available stented part and by performing distal extension as a second-stage procedure. This is in consideration of the fact that the number of simultaneously occluded segmental arteries is only one contributing factor of spinal cord injury (1). Cannulation of the right subclavian artery for arterial return can be performed safely in acute as well as chronic aortic diseases. Aortic arch procedures require complex organ protection and perfusion techniques. In our center, bilateral selective antegrade cerebral perfusion (SACP) is used for cerebral protection, being aware that unilateral SACP is frequently used by other centers in Europe with comparable postoperative stroke rates. The intended perfusate temperature is between 25 and $26^{\circ} \mathrm{C}$ and corresponds to the intended core temperature.

Myocardial protection can be achieved in two different ways. While cold blood cardioplegia in an antegrade as well as retrograde fashion can be effectively used in all scenarios (2), there are selected patients where myocardial protection could be even more effectively achieved by using normothermic selective myocardial perfusion. This is provided that they already have an ascending prosthesis in place, or unaffected native ascending aortic tissue, where cross-clamping is safely feasible. This enables faster myocardial recovery, becoming of cardiopulmonary bypass, and reduces the myocardial injury as well as intraoperative and post-operative catecholamine consumption to a minimum (3). Eventually, combinations can be performed such as root repair during cooling, then switching to a beating-heart concept for aortic arch replacement.

\section{Acute aortic dissection}

The major aim of surgery for acute aortic type A dissection is the survival of the patient. We also know that a key aim of surgery must be the closure of the primary entry tear. If the primary entry tear is located in the mid-ascending aorta and diameters in the aortic arch as well as in downstream aortic segments are regular, hemi-arch replacement is sufficient in most cases. In scenarios where the primary entry tear is located more distally in the aortic arch or proximal descending aorta, which is often associated with a narrow true lumen or even total true lumen collapse resulting in visceral and/or renal malperfusion, surgery that is limited to the ascending aorta may prevent early aortic rupture but leaves the underlying disease mechanism unaddressed. In these cases, the FET technique addresses closure of the primary entry tear as well as expanding the narrow true lumen of the entire aorta to abdominal aortic segments in a domino-type effect (1).

It must be noted that even in patients whose primary entry tear was closed during primary surgery, secondary 
intimal ruptures at the arch anastomosis and either multiple or large communications between the lumina across the membrane are known to pathophysiologically function as a primary entry tear. Consequently, this may also lead to diameter progression or even to a secondary aortic dissection process as an independent event warranting treatment sooner or later. The stent-part of the FET prosthesis reduces the wall stress in the proximal descending aorta and could therefore prevent this secondary aortic dissection process with the need for secondary intervention.

While there is no doubt about the need for surgical therapy of acute type A aortic dissection, treatment options for acute type B and acute non-A non-B aortic dissections are still discussed controversially. Conservative treatment is still a good option for patients with truly uncomplicated type B aortic dissection. However, close follow-up, usually with computed tomography angiography (CTA), as well as optimal medical treatment is warranted. In many cases, the aortic dissection process becomes-per definitioncomplicated by presence of uncontrolled blood pressure or pain, aortic rupture and visceral and/or renal/limb malperfusion. In this scenario, the standard treatment is thoracic endovascular aortic repair (TEVAR) (4). Nevertheless, there are patients with unfavorable aortic anatomy or without an adequate proximal landing zone and would therefore require various degrees of supra-aortic transposition. Whereas a carotid-subclavian bypass or double transposition are well established and should be preferred in these patients, total arch rerouting is associated with a high incidence of retrograde type A aortic dissection (5). In these patients, the FET technique is an attractive method with excellent clinical outcomes and significantly lower risk of a secondary retrograde dissection process (6).

In more than ten percent of patients with an acute aortic dissection, the dissection membrane originates in or extends to the aortic arch, known as non-A non-B aortic dissection. Thereby, the primary entry tear can be located in the aortic arch or descending aorta with retrograde extension, usually as an intramural hematoma (7). In our center, the treatment strategy of complicated aortic dissection with the primary entry tear located in the descending aorta and aortic diameters less than $45 \mathrm{~mm}$, is performed analogously to type $\mathrm{B}$ aortic dissection repair. Alternatively, patients presenting with an arch entry and/or aortic diameters more than 45 $\mathrm{mm}$ are at high risk of retrograde aortic type A dissection, even after previous TEVAR, total aortic arch replacement with the FET technique, is the standard procedure in our institution.

\section{Chronic aortic dissection}

A substantial number of patients require secondary repair due to post-dissection aneurysm formation in primary nontreated aortic segments, after having successfully undergone primary repair of an acute aortic dissection. A few scenarios for the need of aortic reinterventions are fateful; however, many are already predictable at the time of the initial CTA scan, as described previously. Retrospective analyses revealed that one-third of patients had a residual dissection in the aortic arch after previous type A repair, to a variable extent. A residual dissected aortic arch led to aortic growth with the largest diameter increase distal to the left subclavian artery, while the number of communications, the location of a communication at the distal anastomosis as well as falselumen perfusion, were associated with the extent of aortic diameter increase. Most of these patients with "residual" type B aortic dissection after previous type A repair require secondary reinterventions. While endovascular treatment is difficult due to several communications between both lumina and can often narrow true lumen, the FET technique addresses this issue adequately. Narrow true lumina and kinking in the downstream aorta and/or iliac arteries are also frequently seen in patients with chronic aortic diseases. This scenario and in particular, diameter enlargement within the aortic arch, are common in patients with chronic type B aortic dissection, especially in cases initially being treated conservatively or even after previous TEVAR, ultimately requiring total arch replacement during follow-up. Satisfactory remodeling can be achieved by the FET technique due to significant true lumen extension in all aortic segments as well as false-lumen thrombosis, mainly in the thoracic aorta.

In summary, the FET technique has substantially broadened our armamentarium to treat acute and chronic dissection, irrespective of type. The standard application of the method forms the basis of the increasingly relevant "proximal full-fix" strategy and represents an ideal platform for any secondary open or endovascular repair in downstream aortic segments in the years to come.

\section{Acknowledgments}

None.

\section{Footnote}

Conflicts of Interest: The authors have no conflicts of interest 
to declare.

Open Access Statement: This is an Open Access article distributed in accordance with the Creative Commons Attribution-NonCommercial-NoDerivs 4.0 International License (CC BY-NC-ND 4.0), which permits the noncommercial replication and distribution of the article with the strict proviso that no changes or edits are made and the original work is properly cited (including links to both the formal publication through the relevant DOI and the license). See: https://creativecommons.org/licenses/by-nc-nd/4.0/.

\section{References}

1. Berger T, Kreibich M, Morlock J, et al. True-lumen and false-lumen diameter changes in the downstream aorta after frozen elephant trunk implantation. Eur J Cardiothorac Surg 2018;54:375-81.

2. Buckberg GD, Beyersdorf F, Kato NS. Technical considerations and logic of antegrade and retrograde blood cardioplegic delivery. Semin Thorac Cardiovasc Surg 1993;5:125-33.

3. Berger T, Kreibich M, Rylski B, et al. Evaluation of myocardial injury, the need for vasopressors and inotropic support in beating-heart aortic arch surgery. J Cardiovasc Surg (Torino) 2019. [Epub ahead of print].

4. Moulakakis KG, Mylonas SN, Dalainas I, et al. Management of complicated and uncomplicated acute type B dissection. A systematic review and meta-analysis. Ann Cardiothorac Surg 2014;3:234-46.

5. Czerny M, Rieger M, Schmidli J. Incidence, risk factors, and outcome of retrograde type A aortic dissection after TEVAR. Gefässchirurgie 2015;20:45-50.

6. Kreibich M, Berger T, Morlock J, et al. The frozen elephant trunk technique for the treatment of acute complicated Type B aortic dissection. Eur J Cardiothorac Surg 2018;53:525-30.

7. Rylski B, Pérez M, Beyersdorf F, et al. Acute non-A non-B aortic dissection: incidence, treatment and outcome. Eur J Cardiothorac Surg 2017;52:1111-7.

Cite this article as: Berger T, Czerny $M$. The frozen elephant trunk technique in acute and chronic aortic dissection: intraoperative setting and patient selection are key to success. Ann Cardiothorac Surg 2020;9(3):230-232. doi: 10.21037/acs2019-fet-10 